\title{
Double Face Mask Use for COVID-19 Infection Prevention and Control Among Medical Students at Makerere University: A Cross-Section Survey
}

\author{
Racheal Nalunkuma', Derrick Bary Abila ${ }^{1,2}$, Nelson Ssewante (D)', Blaise Kiyimba (D)', Edwin Kigozi $\mathbb{D}^{3}$, \\ Ruth Ketty Kisuza', Fulugensio Kasekende', Jonathan Nkalubo $\mathbb{D}^{\prime}$, Samuel Kalungi ${ }^{4}$, \\ Winters Muttamba $\mathbb{D}^{5}$, Sarah Kiguli ${ }^{6}$
}

'School of Medicine, College of Health Sciences, Makerere University, Kampala, Uganda; ${ }^{2}$ Faculty of Biology, Medicine, and Health, University of Manchester, Manchester, UK; ${ }^{3}$ School of Health Sciences, College of Health Sciences, Makerere University, Kampala, Uganda; ${ }^{4}$ Department of Pathology, Mulago National Referral Hospital, Kampala, Uganda; ${ }^{5}$ Makerere University Lung Institute, Makerere University, Kampala, Uganda; ${ }^{6}$ Department of Paediatrics and Child Health, School of Medicine, Makerere University, Kampala, Uganda

Correspondence: Racheal Nalunkuma, School of Medicine, College of Health Sciences, Makerere University, P.O Box 7072, Kampala, Uganda, Tel +256 704I50697, Email kasoziracheal@gmail.com

Introduction: The second wave of COVID-19 greatly affected the health care and education systems in Uganda, due to the infection itself and the lockdowns instituted. Double masking has been suggested as a safe alternative to double-layered masks, where the quality of the latter may not be guaranteed. This study aimed to determine patterns of double mask use among undergraduate medical students at Makerere University, Uganda.

Methods: We conducted a descriptive cross-sectional study using an online questionnaire. All students enrolled at the College of Health Sciences; Makerere University received the link to this questionnaire to participate. Logistic regression analysis was used to assess factors associated with double mask use.

Results: A total of 348 participants were enrolled. The majority (61.8\%) were male; the median age was 23 (range: 32 ) years. Up to $10.3 \%$, $42 \%$, and $4.3 \%$ reported past COVID-19 positive test, history of COVID-19 symptoms, and having comorbidities, respectively. Up to $40.8 \%$ had been vaccinated against COVID-19. More than half (68.7\%) believed double masking was superior to single masking for COVID-19 IPC, but only $20.5 \%$ reported double masking. Participants with a past COVID-19 positive test [aOR: $2.5 ; 95 \%$ CI: $1.1-5.8, p=0.026$ ] and participants who believed double masks had a superior protective advantage [aOR: 20; 95\% CI: 4.9-86.2, p < 0.001] were more likely to double mask. Lack of trust in the quality of masks (46.5\%) was the most frequent motivation for double masking, while excessive sweating (68.4\%), high cost of masks (66.4\%), and difficulty in breathing (66.1\%) were the major barriers.

Conclusion: Very few medical students practice double masking to prevent COVID-19. Coupled with inconsistencies in the availability of the recommended four-layered masks in Uganda and increased exposure in lecture rooms and clinical rotations, medical students may be at risk of contracting COVID-19.

Keywords: COVID-19, infection prevention and control, double masking, second wave

\section{Introduction}

Coronavirus disease-2019 (COVID-19) was declared a global pandemic on 11th March 2020 by the WHO DirectorGeneral, Dr. Tedros Adhanom Ghebreyesus. ${ }^{1}$ This has been defined as a respiratory disease caused by SARS-CoV-2 that emerged in 2019 in Wuhan, China, and later rapidly spread throughout the world. ${ }^{2}$ This aggressive infection has significantly increased the rates of hospitalization and intensive care unit admissions with a reported mortality rate of about $3-6 \% .^{3}$

As of 17th October 2021, a total of 241,217,863 cases and 4,910,644 deaths have been reported worldwide, while in Uganda, 125,094 cases and 3182 deaths have been reported. ${ }^{4}$ Healthcare workers (HCWs) are one of the categories of people at the highest risk of infection, as they are the first and last line of defense in offering health care services to infected individuals. It 
has been reported that thousands of HCWs have been infected and many have lost their lives. ${ }^{5,6}$ On 7 th July 2021, the Uganda Medical Association reported that Uganda had lost more than 100 health care workers due to COVID-19. ${ }^{7}$

Several infection prevention and control (IPC) measures have been put in place to reduce the spread of COVID-19. These include self-isolation, use of alcohol-based hand sanitizer or hand-washing with soap, restriction of movements with lockdown measures, sanitization of surfaces, and use of face masks or face covering. ${ }^{1,2}$ The Centers for Disease Control and Prevention (CDC) recommended the use of face masks to reduce the spread of COVID-19 infection among individuals. ${ }^{9}$ By covering the nose and mouth, face masks interfere with COVID-19 infection transmission by limiting air spread of respiratory droplets from the infected individual and preventing inhalation of susceptible ones. Some of the recognized types of masks include N95, N100, N99, surgical masks, and cloth masks. ${ }^{8,10}$

An experimental study conducted to demonstrate the efficacy of face masks based on the number of layers revealed that a double-layer mask restricted $91.79 \%$ of droplets as compared to $30.12 \%$ for a single layer. ${ }^{11}$ However, the use of these masks is limited by high costs, availability, and susceptibility to counterfeiting, especially in low resource-limited countries. ${ }^{12}$ To ensure maximal protection, some guidelines recommend double masking, ${ }^{13}$ but this approach is not well documented in our setting and no study has been conducted to evaluate the practice nationally and internationally. Therefore, we set out to determine the patterns of and factors limiting double face mask use practice among undergraduate medical students at the College of Health Sciences, Makerere University, Kampala, Uganda, as a form of IPC during the second wave of the COVID-19 pandemic.

\section{Methods}

\section{Study Design}

Between 5th September 2021 and 21st September 2021, we conducted a descriptive cross-sectional study. This was an online survey recruiting undergraduate medical students at the College of Health Sciences, Makerere University, Kampala, Uganda.

\section{Study Area}

Makerere University, College of Health Sciences (MakCHS) is one of the largest colleges of Makerere University, situated in Kampala, Uganda. The College has four Schools which include the School of Biomedical Sciences, School of Health Sciences, School of Medicine, and School of Public Health, with an estimated population of 1500 undergraduate students.

\section{Target Population}

All undergraduate medical students at MakCHS, 18 years or older, from the first to fifth years of study were targeted. A medical student in this study was defined as one who is enrolled in either of the following courses at Makerere University: Bachelor of Medicine and Surgery (MBChB), Bachelor of Pharmacy (BPHAR), Bachelor of Nursing (BSN), Bachelor of Dental Surgery (BDS), Bachelor of Environmental Health (BEH), and Bachelor of Medical Radiography (BMR), among others.

\section{Sample Size}

The sample size for this study was determined using the Kish Leslie formula for cross-sectional studies.

$$
n=\frac{z^{2} \times p(1-p)}{d^{2}}
$$

where $\mathrm{n}=$ desired sample size, $\mathrm{z}=$ standard deviation ( 1.96 at $95 \%$ confidence interval), $\mathrm{p}=$ estimated to be 0.5 since no previous study had been conducted addressing the same topic, $d=$ acceptable degree of error $(5 \%=0.05), n=385$. To cater for non-respondents, $10 \%$ of the sample size was added; $385+38=423$ students.

\section{Sampling Procedure}

We used the convenience sampling method, whereby those who were able to access the link to the online survey questionnaire sent out were involved. We identified all relevant class WhatsApp and email groups of students through the 
class representative of each year of study. We then continuously shared the invitation link to the online questionnaire via these platforms.

\section{Study Variables}

Independent variables included sex, age, marital status, level of academic progress (clinical vs pre-clinical) and the school and questions on one's COVID-19 vaccination status, signs and symptoms of COVID-19, comorbidity status of participants, perceptions, and barriers. The dependent variable was the use of a double mask for the prevention of COVID-19.

\section{Data Collection Tool}

The questionnaire was adopted from two previous studies ${ }^{14,15}$ and adapted to our study setting. The questionnaire consisted of four sections and was structured as follows:

Section I (sociodemographic characteristics) assessed sex, age, marital status, year of study, and school.

Section II (COVID-19 risk assessment) assessed participants' vaccination status, history of positive COVID-19 tests, signs and symptoms, and comorbidities of COVID-19.

Section III (face masking practices) assessed common types of masks used among participants, double masking practices, combinations commonly used, and motivation factors for double masking.

Section IV (barriers to double masking) assessed the factors limiting double mask use.

\section{Quality Control}

Questions were designed in simple English words for effective understanding by the participants. The questionnaire was designed with validation checks and skip logics to ensure quality and complete entries. The principal investigator directly supervised all the activities from the beginning to the completion of the study.

\section{Data Management and Analyses}

Data was collected using Kobo Toolbox software. Completed data were exported to Microsoft Excel 2016 for cleaning and coding. After that, this was imported into STATA 15 for formal analysis. All categorical variables were summarized as frequencies and proportions, while median age was calculated. The association between double masking and predictors (sociodemographic characteristics, COVID-19 infection, and vaccination status) was assessed using Chi-square/Fischer's exact test, and logistic regression model was used to calculate the adjusted odds ratios (aORs) of the predictor variables concerning double face mask use. A p-value less than 0.05 was considered statistically significant.

\section{Ethical Considerations}

The study was performed according to the Declaration of Helsinki, after obtaining approval from Mulago Hospital Research and Ethics Committee (Reference number: MHREC 2114). Mulago is a teaching hospital for the College of Health Sciences, Makerere University. Participation in the study was entirely voluntary, and the participants were required to consent by clicking on the "I accept to participate" button at the end of the consent statement attached to the questionnaire before they started answering the questions.

\section{Results}

\section{Characteristics of Participants}

Table 1 represents the patient characteristics. A total of 348 (82.3\% response rate) responses were collected and included in the analysis. Of these, the majority (61.8\%) were males, and the overall median age was 23 (range: 32 ) years. More than half (57.5\%) were in clinical years of study (year 3 and above) and were from the School of Medicine (56.9\%).

Of the surveyed participants, $40.8 \%$ had been vaccinated, $10.3 \%$ reported the previous history of COVID-19, and $42 \%$ reported experiencing COVID-19 signs and symptoms. Up to $4.3 \%$ of participants reported having comorbidities 
Table I Characteristics and Factors Associated with Double Masking Among Study Participants

\begin{tabular}{|c|c|c|c|c|}
\hline \multirow[t]{2}{*}{ Variable } & \multirow[t]{2}{*}{ Total } & \multicolumn{3}{|c|}{ Double Masking } \\
\hline & & No, n (\%) & Yes, n (\%) & p-value \\
\hline Overall & & $275(79.5)$ & $71(20.5)$ & \\
\hline Age [median: 23 (range: 32)] & & & & 0.695 \\
\hline$>23$ years & | 44 (4I.4) & II $3(78.5)$ & $31(21.5)$ & \\
\hline$\leq 23$ years & $204(58.6)$ & $162(80.2)$ & $40(19.8)$ & \\
\hline Sex & & & & 0.105 \\
\hline Female & $133(38.2)$ & $99(75)$ & $33(25)$ & \\
\hline Male & $215(61.8)$ & $176(82.2)$ & $38(17.8)$ & \\
\hline Academic progress & & & & 0.108 \\
\hline Clinical & $200(57.5)$ & $153(76.5)$ & $47(23.5)$ & \\
\hline Pre-clinical & $148(42.5)$ & $122(83.6)$ & $24(16.4)$ & \\
\hline School & & & & 0.327 \\
\hline School of Biomedical Sciences & $36(10.3)$ & $29(80.6)$ & $7(19.4)$ & \\
\hline School of Health Sciences & $98(28.2)$ & 81 (82.7) & $17(17.3)$ & \\
\hline School of Medicine & $198(56.9)$ & $150(76.5)$ & $46(23.5)$ & \\
\hline School of Public Health & $16(4.6)$ & $15(93.8)$ & I (6.3) & \\
\hline Marital status & & & & 0.244 \\
\hline Married & $42(12.1)$ & $30(71.4)$ & $12(28.6)$ & \\
\hline Prefer not to say & $20(5.8)$ & $18(90)$ & $2(10)$ & \\
\hline Single & $286(82.2)$ & $227(79.9)$ & $57(20.1)$ & \\
\hline COVID-19 vaccination status & & & & 0.188 \\
\hline Not vaccinated & $206(59.2)$ & $167(81.9)$ & $37(18.1)$ & \\
\hline Vaccinated & $142(40.8)$ & $108(76.1)$ & $34(23.9)$ & \\
\hline History of positive COVID-19 test & & & & 0.003 \\
\hline No & $312(89.7)$ & $254(81.7)$ & $57(18.3)$ & \\
\hline Yes & $36(10.3)$ & $21(60)$ & $14(40)$ & \\
\hline History of COVID-I 9 symptoms & & & & 0.346 \\
\hline No & $|8|(58)$ & $|5|(83.4)$ & $30(16.6)$ & \\
\hline Yes & $|3|(42)$ & $103(79.2)$ & $27(20.8)$ & \\
\hline COVID-I 9 comorbidities & & & & 0.499 \\
\hline No & $331(95.7)$ & $263(79.7)$ & $67(20.3)$ & \\
\hline Yes & $15(4.3)$ & $10(71.4)$ & $4(28.6)$ & \\
\hline Double masking versus single mask & & & & $<0.001$ \\
\hline Similar & $108(31.3)$ & $106(98.1)$ & $2(1.9)$ & \\
\hline Double masking > single mask & $237(68.7)$ & $168(70.9)$ & $69(29.1)$ & \\
\hline
\end{tabular}

including hypertension, diabetes mellitus, and asthma, among others (Figure 1). More than half (68.7\%) believed double masking was superior to single masking for IPC.

\section{Face Mask Practices Among Participants}

The face mask practices among the participants are indicated in Table 2. More than half of the participants (68.6\%) believed double masking was superior to single masking, with less than a third (20.5\%) reporting they were double masking. The most common double masking combination was two surgical masks (68.8\%), with the majority doing so 


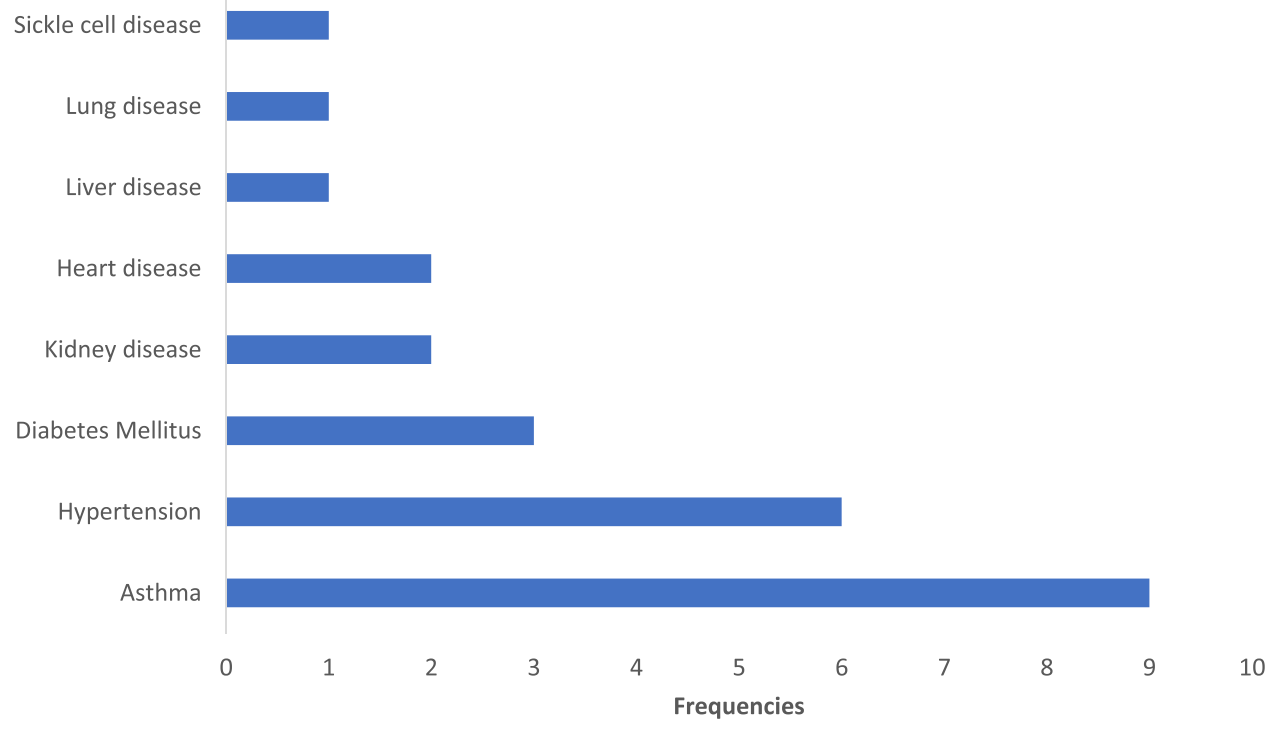

Figure I Common COVID-19 comorbidities among study participants.

while in the hospital wards $(62.0 \%)$. The most frequently reported motivation for double masking was a lack of trust in the quality of the masks $(46.5 \%)$.

\section{Factors Associated with Double Masking Practices}

As shown in Table 1, a history of previous COVID-19 infection $(\mathrm{p}=0.003)$ and knowledge of the superiority of double masking relative to single masking $(\mathrm{p}<0.001)$ were associated with double masking.

Table 2 FaceMasks Practices and Motivation Factors for Double Masking

\begin{tabular}{|l|l|l|}
\hline Variable & Frequency & Percentages \\
\hline Face masking practices & & \\
Single mask & 275 & 79.5 \\
Double mask & 71 & 20.5 \\
\hline When do you double mask & & \\
On the ward & 44 & 62.0 \\
In a public place & 39 & 54.9 \\
In a lecture room & 10 & 14.1 \\
\hline Mask combinations & & \\
Two surgical masks & 55 & 77.5 \\
Two cloth masks & 3 & 4.2 \\
N95 and a surgical mask & 8 & 11.3 \\
KN95 and a surgical mask & 5 & 7.0 \\
Surgical mask and a respirator & 1 & 1.4 \\
Surgical masks and a cloth mask & 5 & 7.0 \\
\hline Motivating factors to double masks & & \\
To ensure tightness & 31 & 43.7 \\
I do not trust the quality of masks & 33 & 46.5 \\
University requirement & 8 & 11.3 \\
It offers more protection & 7 & 9.9 \\
\hline
\end{tabular}


Table 3 Multivariate Logistic Regression of the Factors Associated with Double Masking

\begin{tabular}{|c|c|c|}
\hline Variables & aOR (95\% Cl) & p-values \\
\hline \multicolumn{3}{|l|}{ Sex } \\
\hline \multicolumn{3}{|l|}{ Male } \\
\hline Female & $1.3(0.8-2.4)$ & 0.323 \\
\hline \multicolumn{3}{|l|}{ Academic progress } \\
\hline \multicolumn{3}{|l|}{ Pre-clinical } \\
\hline Clinical & $1.2(0.6-2.1)$ & 0.622 \\
\hline \multicolumn{3}{|l|}{ Vaccination status } \\
\hline \multicolumn{3}{|l|}{ Not vaccinated } \\
\hline Vaccinated & $1.6(0.9-2.8)$ & 0.107 \\
\hline \multicolumn{3}{|c|}{ History of positive COVID-I 9 test } \\
\hline \multicolumn{3}{|l|}{ No } \\
\hline Yes & $2.5(1.1-5.8)$ & 0.026 \\
\hline \multicolumn{3}{|l|}{ COVID-19 comorbidities } \\
\hline \multicolumn{3}{|l|}{ No } \\
\hline Yes & I.4 (0.4-5.3) & 0.613 \\
\hline \multicolumn{3}{|c|}{ Double masking versus single mask } \\
\hline \multicolumn{3}{|l|}{ Similar } \\
\hline Double masking $>$ single mask & $20.6(4.9-86.2)$ & $<0.001$ \\
\hline
\end{tabular}

Notes: Cox-Snell R-squared: 0.146, Nagelkerke R-squared: 0.228 , model p: $<0.00$ I constant p: $<0.001$ Abbreviations: aOR, adjusted odds ratio; $95 \% \mathrm{Cl}, 95 \%$ confidence interval.

Participants who had a history of COVID-19 infection [aOR: 2.5; 95\% CI: 1.1-5.8, $\mathrm{p}=0.026$ ] were more likely to double mask compared to those who had never been diagnosed with COVID-19. Likewise, participants who believed double masks had a superior protective advantage were more likely to double mask [aOR: 20; 95\% CI: 4.9-86.2, p < 0.001] (Table 3).

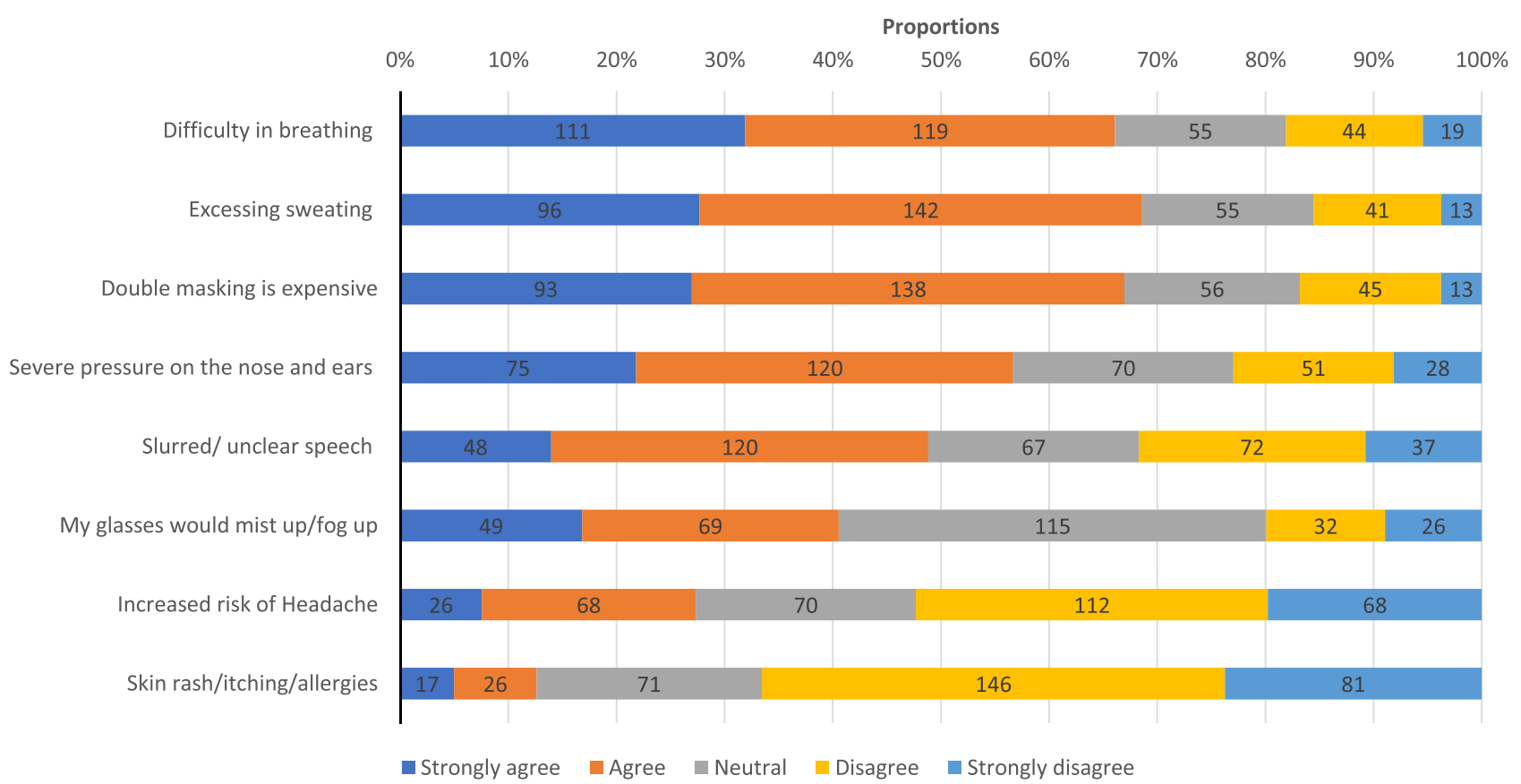

Figure 2 Barriers to double face mask use among study participants. 


\section{Barriers to Double Masking Use}

Figure 2 summarizes the reported barriers to double masking among the respondents. Excessive sweating (68.4\%), high cost of masks $(66.4 \%)$, and difficulty in breathing $(66.1 \%)$ were frequently reported as the major barriers to double masking.

\section{Discussion}

Worldwide, the health care system has been affected by the COVID-19 pandemic. However, the discovery of vaccines that protect against severe forms of COVID-19 is seen as a great innovation that has contributed to a significant reduction in mortality associated with this disease. Unfortunately, to date, the vaccination coverage among resource-limited countries including Uganda is still way below the recommended levels to achieve herd immunity. ${ }^{16}$ For that reason, Uganda still depends majorly on standard operating procedures (SOPs) that break the inter-personal transmission of infections such as social distancing and routine wearing of face masks. Throughout the pandemic, several face masks were designed and promoted to control infection transmission. However, this came along with it several counterfeit products, especially in resource-limited countries where the costs of standard masks may be unaffordable to the population. ${ }^{12}$ This puts this susceptible population at risk of contracting COVID-19. Medical students across the medical schools in Uganda were allowed to resume school activities with an emphasis on SOPs and before being fully vaccinated against COVID-19. Given the exposure medical students get during their clinical rotations, these SOPs including ensuring maximal effectiveness of masking practices are key to preventing the risk of acquiring COVID-19. In the current study, we found a very low rate of double mask use although participants knew the superiority of double masking as compared to single masking. Additionally, the vaccination status was still low among the students.

Our study showed that more than half of participants believed double masking was more protective than a single mask, but less than a third double-masked to protect themselves from COVID-19. Notably, the majority of those practicing double masking were combining either surgical masks or a surgical mask and either N95 or KN95. According to the Centers for Disease Prevention and Control (CDC), double masking significantly reduces the risk of infection transmission to $85.4 \%$ using a surgical mask in combination with a cloth mask. ${ }^{17}$ This is also supported by the quality improvement study conducted by the University of North Carolina that showed that wearing a medical procedure mask underneath a cloth mask provided the best improvement to fitted filtration efficiency (FFE) of all the combinations evaluated. ${ }^{13,18}$ This is particularly important in a highly infectious environment such as in the hospital environment and public places. ${ }^{19}$ The low practice of combining a surgical mask and a cloth mask among our participants is an indicator that there exists a knowledge gap and they are limited in their choices for which approach to use. A rich pool of varying guidelines on the best type of masks discourages the use of single cloth masks among health workers due to their low filtration efficiency, which could have limited its selection in double mask combinations among our participants. ${ }^{20,21}$

Alternative use of a single N95 or KN95 mask has been backed by the CDC as a sufficient approach that can offer desired protection against COVID-19, ${ }^{16}$ but the inconsistencies in the quality of these masks on the market have been a big threat to their recommendation. The question of quality masks on the market is especially likely to be of significance in developing countries like Uganda where high costs of the effective N95/KN95 may be a driving factor in the promotion of cheaper but counterfeit products. This was evident in our study with most of our participants reporting that the most important factor leading to double masking was mistrust in the quality of masks. Since most participants had a preference towards combining a surgical mask with either another surgical mask or N95/KN95 that are generally expensive, it could have affected their ability to routinely double mask regardless of the known benefit. The majority of the participants reported double masks while on the wards. This is in line with recommendations from a study conducted at Zhongnan Hospital in China, for all medical staff to wear protective suits and double masks to reduce the likelihood of acquiring COVID-19. ${ }^{22}$ A hospital setting predisposes one to COVID-19 partly by being a collection center for people from all over and the increased likelihood that these people are infected. Participants in the current study though are frequently exposed to a hospital environment due to the nature of their training, little has been done to ensure their safety. This is evidenced by the fact that although by the time this study was conducted, Uganda was in a second wave of COVID-19 with a high infectivity rate, only $40.8 \%$ of participants had received at least one shot of the vaccine. The risk of COVID-19 mortality among the unvaccinated population cannot be overemphasized. ${ }^{22,23}$ 
The high costs of face masks, difficulty in breathing, excessive sweating, and excessive pressure were the main barriers reported by participants to double masking in the current study. In 2016, it was estimated that up to $41.7 \%$ of Ugandans were below the poverty line (relying on $<\$ 1.9$ per day which is approximately $<7000$ Uganda shillings). ${ }^{24}$ This is even worse in the ongoing COVID-19 lockdown which has slowed down the economy. With a single non-reusable surgical mask costing 2000 Ugandan shillings, it is not surprising that very many Ugandans would not afford to double mask, especially when the practice is restricted to the non-reusable type of masks. Generally, face masks are discomforting, and according to the WHO, they should not be worn beyond 4 hours as beyond this level wearers become restless and may lead to self-contamination. ${ }^{25}$ The level of discomfort is worsened with double masking. Caution must therefore be taken while selecting face masks to choose the perfect fitting to ensure comfort and tolerability. Although there is some published evidence that increased tightness which might be a result of double masking may be a predisposing factor for adverse skin reactions, in the current study, this was of very low impact in our study. ${ }^{26}$ In fact, most participants in our study aimed at improving the tightness of masks to achieve maximal protection.

\section{Strength of the Study}

This study is the first of its kind in our setting. Given the low rate of vaccine coverage, Uganda still faces a threat of further COVID-19 waves. This study provides important information that can be used to strengthen face masking policies to maximize protection and reduce COVID-19 transmission.

\section{Limitations}

This study was purely online due to COVID-19 restrictions. Therefore, potential participants who did not have supporting gadgets or poor connectivity to the internet or those who could not afford data costs for participating in this study were excluded. Since the questionnaire was self-administered, participants may have incorrectly reported double face mask use in fear of being blamed for not following SOPs. The study focussed on medical students who may be better informed compared to the general population as regards COVID-19 SOPs which may affect the generalization of findings. Finally, in addition to the small sample size and the variability in the social determinants of health across the different continents, these findings may not be generalizable outside the African continent.

\section{Conclusion}

Very few medical students practice double masking to prevent COVID-19. Coupled with inconsistencies in the availability of the recommended four-layered masks in Uganda and increased exposure in lecture rooms and clinical rotations, medical students may be at risk of contracting COVID-19. A cheaper alternative with a combination of a surgical and cloth mask should be promoted as a COVID-19 IPC measure. More population studies need to be conducted to inform policy development.

\section{Acknowledgments}

The authors would like to thank the Lord God Almighty for His never-ending grace. The principal investigator wishes to express sincere gratitude to Prof. Henry Kasozi, Mrs. Sayuni Kasozi, Mr. Fred Kasozi, Mrs. Oliver Kasozi, Ms. Aida Kasozi, Mr. Richard Kabaale Kasozi, Mrs. Ritah Nansubuga, Robert Kasozi, Mrs. Florence Kasozi M, Adrianna Naomi Sanyu Nanono, Dr. Male Mutumba and the entire K family for the encouragement and support they have offered towards my career development. The authors also appreciate Ms. Evelyn Bakengesa, Ms. Regina, and Ms. Rhoda from HEPISHSSU for their support rendered towards the acquisition of funds.

\section{Funding}

Research reported in this publication was supported by the Fogarty International Center of the National Institutes of Health, US Department of State's Office of the US Global AIDS Coordinator and Health Diplomacy (S/GAC), and President's Emergency Plan for AIDS Relief (PEPFAR) under Award Number 1R25TW011213. The content is solely the responsibility of the authors and does not necessarily represent the official views of the National Institutes of Health. 


\section{Disclosure}

The authors report no conflicts of interest in this work.

\section{References}

1. Cucinotta D, Vanelli M. WHO declares COVID-19 a pandemic. Acta Bio-Medica. 2020;91(1):157-160. doi:10.23750/abm.v91i1.9397

2. Lin YCC, Tsai M, Lu CHP, Hou M. Infection control measures of a Taiwanese hospital to confront the COVID-19 pandemic. Kaohsiung J Med Sci. 2020;36:296-304.

3. Barranco R, Ventura F. COVID-19 and infection in health-care workers: an emerging problem. The silent deaths of the elderly in long-term care facilities during the Covid-19 pandemic: the role of forensic pathology. Medico Legal J. 2020;88(2):65-66. doi:10.1177/0025817220923694

4. Worldometers. Coronavirus Updates. Worldmeter; 2021.

5. Lake MA. What we know so far: COVID-19 current clinical knowledge and research. Clin Med. 2020;20:124-127. doi:10.7861/clinmed.2019coron

6. Koh D, Goh HP. Occupational health responses to COVID-19: what lessons can we learn from SARS? J Occup Health. 2020;62(1):e12128. doi:10.1002/1348-9585.12128

7. VOA. Uganda Loses 37 Health Workers to COVID-19. VOA; 2021.

8. Katembo F, Id S, Id RS, et al. Use of face masks to limit the spread of the COVID-19 among western Ugandans: knowledge, attitude and practices. PLoS One. 2021;16:1-13.

9. Brooks JT, Beezhold DH, Noti JD, Coyle JP, Derk RC, Blachere FM. Maximizing fit for cloth and medical procedure masks to improve performance and reduce SARS-CoV-2 transmission and exposure, 2021. Gut. 2021;70(7):2019-2022. doi:10.1136/gutjnl-2021-324112

10. Stutt RO, Retkute R, Gilligan CA, Colvin J. A modeling framework to assess the likely effectiveness of facemasks in combination with ' lock-down ' in managing the COVID-19 pandemic subject areas. Proc Royal Soc A. 2020;476:20200376.

11. Sharma S, Pinto R, Saha A, Chaudhuri S, Basu S. On secondary atomization and blockage of surrogate cough droplets in single- and multilayer face masks. Sci Adv. 2021;7:1-12.

12. Plana D, Tian E, Cramer AK, et al. Assessing the filtration efficiency and regulatory status of N95s and nontraditional filtering face-piece respirators available during the COVID-19 pandemic. BMC Infect Dis. 2021;21(1):1-13. doi:10.1186/s12879-021-06008-8

13. Stephenson J. CDC studies underscore continued importance of masks to prevent Coronavirus spread. Am Med Assoc. 2021;2(2):1-2.

14. Macintyre CR, Wang Q, Cauchemez S, Seale H, Dwyer DE. A cluster randomized clinical trial comparing fit-tested and non-fit-tested N95 respirators to medical masks to prevent respiratory virus infection in health care workers. Influenza Other Respir Viruses. 2011;5:170-179. doi:10.1111/j.1750-2659.2011.00198.x

15. Matusiak Ł, Szepietowska M, Krajewski P, Birula RB, Jacek C. Inconveniences due to the use of face masks during the COVID-19 pandemic: a survey study of 876 young people. Dermatol Ther. 2020;33. doi:10.1111/dth.13567

16. Musoke P, Okot J, Nanfuka V, et al. A preliminary report on herbal medicine use among patients hospitalized at two-large COVID-19 treatment centers in Uganda. Risk Manag Healthc Policy. 2021;14:4609. doi:10.2147/RMHP.S339408

17. Brooks JT, Beezhold DH, Noti JD. Maximizing fit for cloth and medical procedure masks to improve performance and reduce SARS-CoV-2 transmission and exposure, 2021. MMWR Morb Mortal Wkly Rep. 2021;70(7):254-257. doi:10.15585/mmwr.mm7007e1

18. Sickbert-Bennett EE, Samet JM, Prince SE, et al. Fitted filtration efficiency of double masking during the COVID-19 pandemic. JAMA Intern Med. 2021;181(8):1126-1128. doi:10.1001/jamainternmed.2021.2033

19. Rothamer DA, Sanders S, Reindl D, Bertram TH. Strategies to minimize SARS-CoV-2 transmission in classroom settings: combined impacts of ventilation and mask effective filtration efficiency. medRxiv. 2021. doi:10.1101/2020.12.31.20249101v1

20. Scalvenzi M, Villani A, Ruggiero A. Community knowledge about the use, reuse, disinfection and disposal of masks and filtering facepiece respirators: results of a study conducted in a dermatology clinic at the University of Naples in Italy. J Commun Health. 2021;46(4):786-793. doi:10.1007/s10900-020-00952-3

21. Chughtaita AA, Seale H, MacIntyre CR. Effectiveness of cloth masks for protection against severe acute respiratory syndrome coronavirus 2. Emerg Infect Dis. 2020;26(10). Available from: https://pubmed.ncbi.nlm.nih.gov/32639930/.

22. Liu C. Antibodies in infants born to mothers with COVID-19 pneumonia tests for IgG and IgM antibodies for severe acute respiratory training and fit testing of health care personnel for reusable elastomeric half-mask respirators compared with disposable N95 respirators. The demand for disposable respiratory protective devices. JAMA. 2020;323(18):2020-2021.

23. Dyer O. Covid-19: unvaccinated face 11 times risk of death from delta variant, CDC data show. BMJ. 2021;374:n2282. doi:10.1136/bmj.n2282

24. Owori M. Poverty in Uganda: national and regional data and trends - development initiatives. Development Initiatives Factsheet; 2020. Available from: https://devinit.org/resources/poverty-uganda-national-and-regional-data-and-trends/\#note-source-CAmD844bm. Accessed January 15, 2022.

25. World Health Organisation. Rational use of personal protective equipment (PPE) for coronavirus disease (COVID-19) - interim guidance (19 March 2020) - World | reliefWeb [Internet]. Manual and Guideline; 2020. Available from: https://reliefweb.int/report/world/rational-usepersonal-protective-equipment-ppe-coronavirus-disease-covid-19-interim. Accessed January 15, 2022.

26. Christopher PM, Roren RS, Tania C, Jayadi NN, Cucunawangsih C. Adverse skin reactions to personal protective equipment among health-care workers during COVID-19 pandemic: a multicenter cross-sectional study in Indonesia. Int J Dermatol Venereol. 2020;3:211-218. doi:10.1097/ JD9.0000000000000132 


\section{Publish your work in this journal}

Risk Management and Healthcare Policy is an international, peer-reviewed, open access journal focusing on all aspects of public health, policy, and preventative measures to promote good health and improve morbidity and mortality in the population. The journal welcomes submitted papers covering original research, basic science, clinical \& epidemiological studies, reviews and evaluations, guidelines, expert opinion and commentary, case reports and extended reports. The manuscript management system is completely online and includes a very quick and fair peer-review system, which is all easy to use. Visit http://www.dovepress.com/testimonials.php to read real quotes from published authors.

Submit your manuscript here: https://www.dovepress.com/risk-management-and-healthcare-policy-journal 